\title{
Archéopages
}

Archéopages

Archéologie et société

\section{La restitution 3D comme outil de recherche archéologique. L'exemple du bâtiment antique de Bouxières-sous-Froidmont}

Karine Boulanger

\section{(2) OpenEdition \\ Journals}

Édition électronique

URL : https://journals.openedition.org/archeopages/317

DOI : 10.4000/archeopages.317

ISSN : 2269-9872

Éditeur

INRAP - Institut national de recherches archéologiques préventives

Édition imprimée

Date de publication : 1 mai 2013

Pagination : 87-89

ISSN : 1622-8545

Référence électronique

Karine Boulanger, «La restitution 3D comme outil de recherche archéologique. L'exemple du bâtiment antique de Bouxières-sous-Froidmont », Archéopages [En ligne], 35 | 10/2012, mis en ligne le 01 octobre 2014, consulté le 25 janvier 2022. URL : http://journals.openedition.org/archeopages/317 ; DOI : https://doi.org/10.4000/archeopages.317 
un puissant outil de compréhension des ensembles bâtis complexes ou disparus, grâce aux possibilités offertes: la visualisation à partir de différents points de vue, l'extraction de coupes ou de vues écorchées, et la possibilité d'ajouter ou de masquer des structures appartenant à différentes phases de construction. La $3 \mathrm{D}$ permet également une présentation pédagogique au public de vestiges disparus ou inaccessibles, notamment par les bornes interactives dans les programmes muséographiques ou sur les sites patrimoniaux accessibles via internet.

Le sous-investissement des archéologues dans la $3 \mathrm{D}$ tient peut-être au fait que les relevés sont réalisés par des cabinets spécialisés. Dès lors, les archéologues risquent de se voir marginalisés face à la tendance du «tout technique » de ces cabinets d'ingénieurs, capables de fournir des belles images mais sans réelle valeur archéologique ou historique car créées sans analyse technique et stratigraphique. Or ces démarches constituent justement la particularité de l'archéologie par rapport aux autres métiers du patrimoine. Les clients de ces services de $3 \mathrm{D}$, essentiellement des collectivités, sont-ils suffisamment informés pour connaître les limites des méthodes et du produit fourni? Lappropriation de ces techniques par les archéologues permettra d'assurer la validité des restitutions et de constituer une archive numérique évolutive. Les chantiers préventifs ont d'ores et déjà démontré les apports de l'archéologie dans le domaine du patrimoine bâti.

\section{Références bibliographiques}

BARBER D., MiLLS J., 2007, 3 D laser scanning for heritage. Advice and guidance to users on laser scanning in archaeology and architecture, English Heritage, Londres.

BöHLER W., HeInz G., Marbs A., 2004, « The Potential of Non-Contact Close Range Laser Scanners for Heritage Recording Purposes », in Geoinformatics 2004, Proceedings of the $12^{\text {th }}$ International Conference on Geoinformatics. Geospatial Information Research: Bridging the Pacific and Atlantic, Actes du colloque de l'Universitéde Gävle (Suède), 7-9 Juin 2004, p. 291-298.

BRYANT S., 2007, Le Grand-Pressigny, 37.113.034.AH, Indre-et-Loire, Région Centre. Le nymphée du château. Une architecture et un système hydraulique de la Renaissance, Rapport de diagnostic archéologique, Inrap, Orléans.

Bryant S., Aubourg V. (collab.), Philippe M. (collab.), WEDAJO B. (COLLAB.), 2010, Blois (41 - Loir-et-Cher), le Château. Programme d'opération archéologique pluriannuelle, campagne 2010. Campagne de relevés archéologiques des élévations de la tour de Foix et du bastion dit de Marie de Médicis, rapport archéologique, Inrap, Orléans.

Bryant S., Boukef Y. (collab.), Prevot A. (Collab.), WEDAJO B. (COLLAB.), 2012, Blois (41 - Loir-et-Cher), le Château. Les latrines de Louis XII, première approche archéologique des élévations, rapport d'étude archéologique, Inrap, Orléans.

Buehrer Th., Fraser C., Gruen A., Li Z., Ruther H., 2001, « Photogrammetric reconstruction and ${ }_{3} \mathrm{D}$ visualisation of Bet Giorgis, a rock-hewn church in Ethiopia ", in Proceedings of the XVIII CIPA Symposium, Postdam, 18 -21 Septembre 2001, article en ligne, http://cipa.icomos.org/text\%2ofiles/ potsdam/2001-07-tbo2.pdf, consulté le 14 mars 2001

Coordination: Sylvie Deffressigne, Inrap. 2. Inrap, architecte de formation.

3. Étudiant à l'école nationale supérieure d'Architecture de Nancy.
La restitution $3 \mathrm{D}$ comme outil de recherche archéologique.

L'exemple du bâtiment antique de Bouxières-sous-Froidmont

Karine Boulanger, Inrap, EA 1132

« Histoire et cultures de l'Antiquité et du Moyen Âge »

En archéologie, la restitution 3D n'est pas exclusivement réservée à la conception d'illustrations pédagogiques esthétiques destinées à la valorisation des sites auprès du grand public. Elle peut également être conçue comme un outil essentiel à l'approfondissement de la réflexion des chercheurs. L'expérience menée à Bouxières-sous-Froidmont (Meurthe-et-Moselle), au lieu-dit «Le Tremble», témoigne de l'intérêt de cette démarche.

Cette fouille archéologique préventive, réalisée en 2003 , a livré un établissement rural isolé dont l'occupation s'échelonne entre le milieu du i ${ }^{\mathrm{er}}$ et le milieu du $\mathrm{III}^{\mathrm{e}}$ siècle. Ce bâtiment sur fondations en pierre bénéficiait de niveaux de sol bien conservés. Létude des vestiges a notamment permis de caractériser les différents espaces et de préciser la nature des matériaux et des techniques constructives des élévations [cf. article p. 34-41]. Dans l'espace interne de la grange, pièce principale de la construction, la présence de trous de poteaux a particulièrement retenu l'attention des fouilleurs [ill. 1]. Ces indices d'une structure porteuse, contemporaine des murs maçonnés, ont ouvert le débat sur les différentes possibilités de restitution des élévations. Afin de pousser la réflexion à son terme et démontrer la compatibilité d'un bâtiment sur fondations en pierre avec une structure porteuse sur poteaux de bois, nous avons tenté l'exercice d'une restitution 3D. Cette expérience a été rendue possible grâce à la collaboration de René Elter ${ }^{2}$ et à la participation bénévole de Sylvain Poilprez ${ }^{3}$.

Dans un premier temps, le travail de restitution a été guidé par la mise en évidence des schémas régulateurs régissant le plan de l'édifice. La grange et ses aménagements internes sont tracés à partir d'un module carré de base, de 10 pieds de côté [ill. 1, tracé vert]. Le développement des espaces périphériques postérieurs est quant à lui basé sur le report d'un module rectangulaire correspondant à la moitié de la superficie de la construction initiale [ill. 1, tracé rouge]. Dans un second temps, il est apparu que la restitution d'élévations en pan de bois sur soubassement maçonné était la plus appropriée à notre cas de figure [ill. 2]. En effet, les poteaux internes semblent destinés à soutenir le plancher d'un étage conçu comme une structure indépendante, partiellement liée aux élévations de la grange [ill. 3]. Cette méthode constructive suggère que les murs périphériques sont réalisés en structure légère. L'existence de parois en torchis est par ailleurs suggérée par la découverte de couches d'argile crue et de torchis brûlé dans les niveaux d'abandon du bâtiment. Le choix de la hauteur des élévations 
1.Plan général du bâtiment et de ses tracés régulateurs. Deux établissements antiques similaires ont été fouillés dans une aire géographique d'un rayon de $10 \mathrm{~km}$ : La Grangeaux-Bois et Metz Queuleu

(Moselle). Ces constructions "I standardisées ॥, d'une superficie d'environ $150 \mathrm{~m}^{2}$,

correspondent à des fermes isolées, adaptées aux besoins d'une unité familiale pour l'exploitation agropastorale d'un modeste terroir.Sont représentés en vert la grange et ses aménagements, en rouge les espaces périphériques.

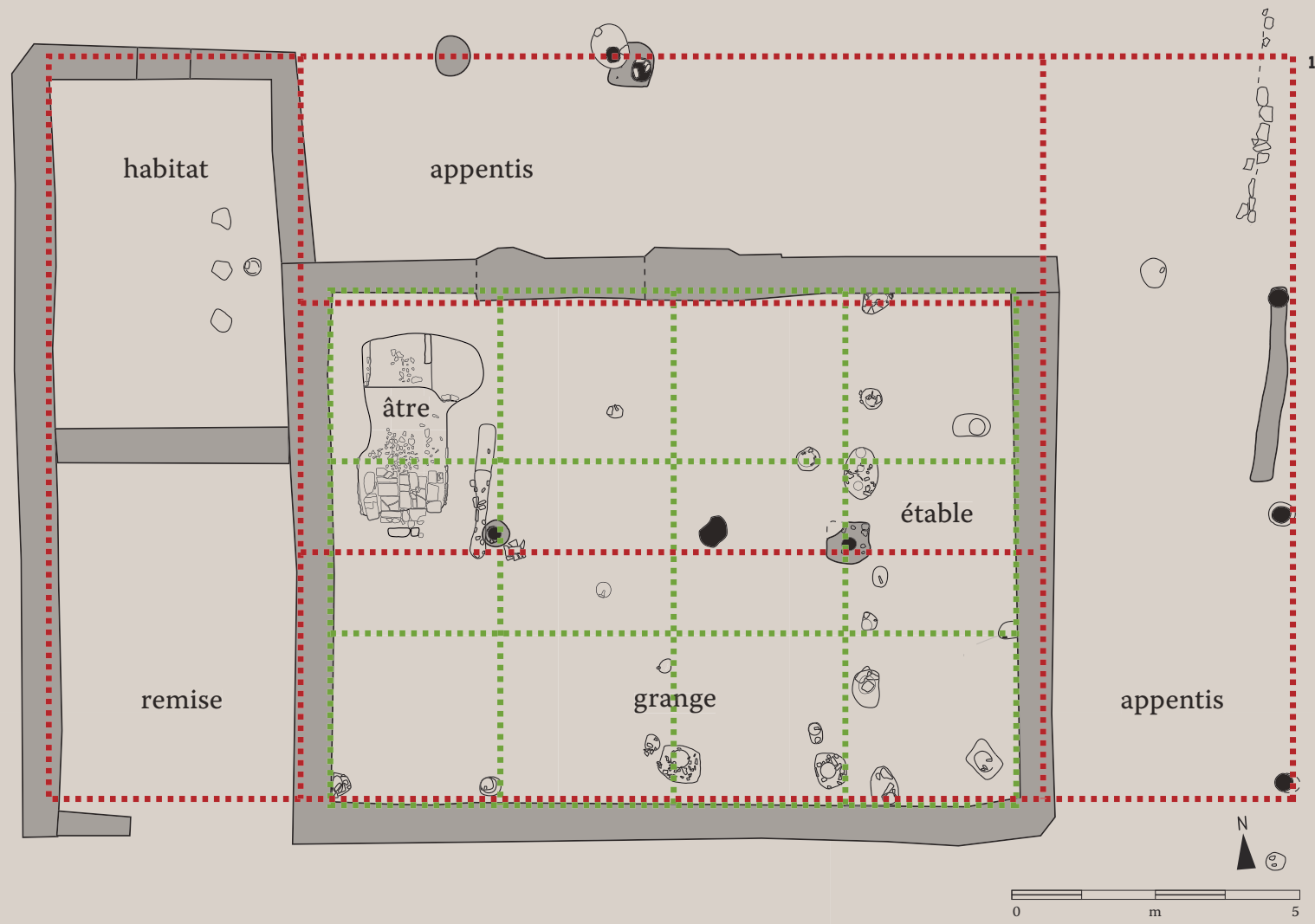

2.a

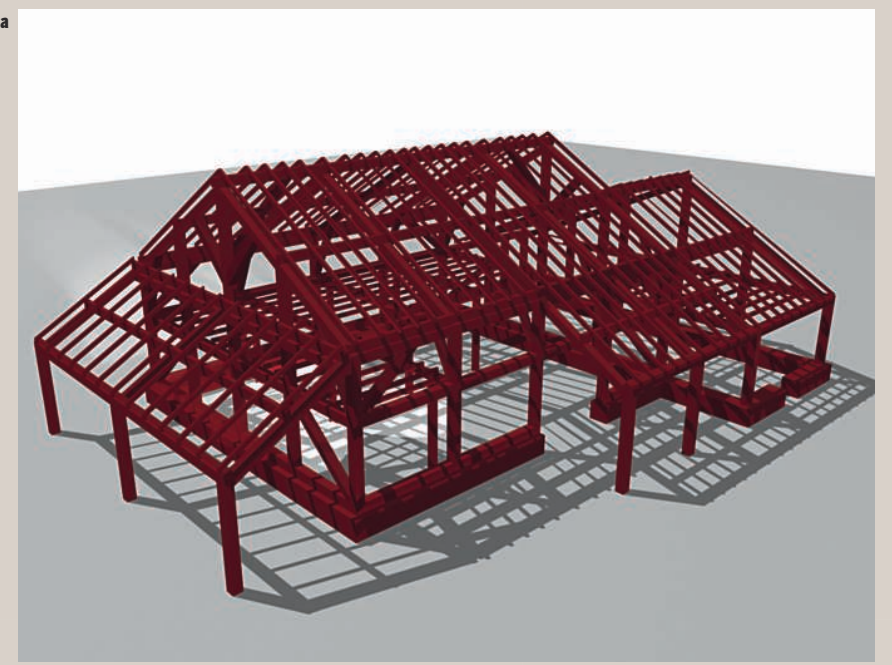

2.a,bet c. Deux étapes du processus de restitution $3 D$ du bâtiment avec vues des façades avant et latérale. Ces images ayant été initialement conçues comme des documents de travail,

des évocations des élévations du bâtiment de ferme, les habillages et les textures n'ont pas été traitées dans le détail.

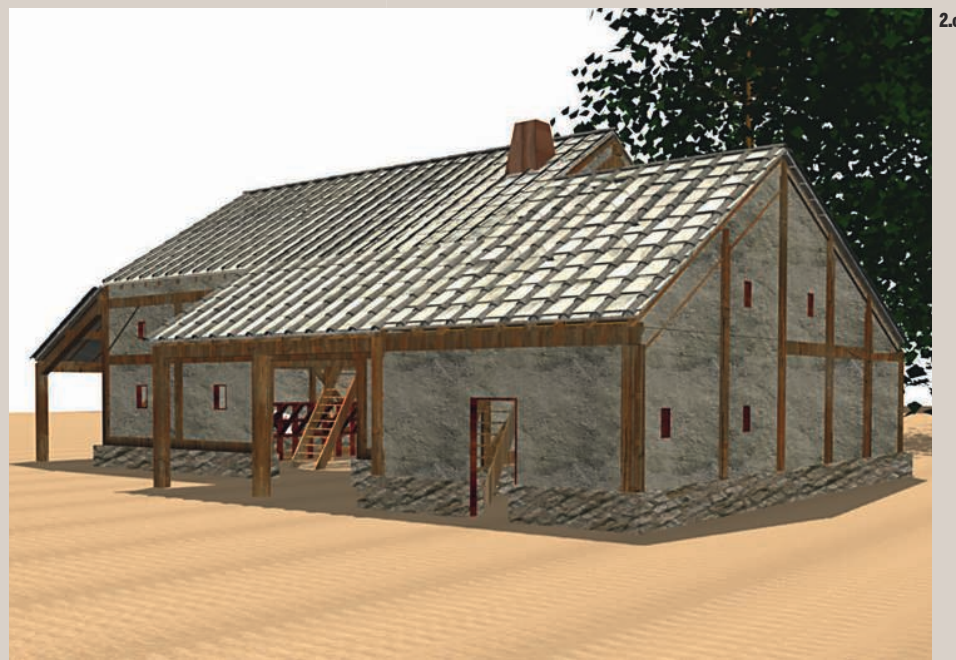

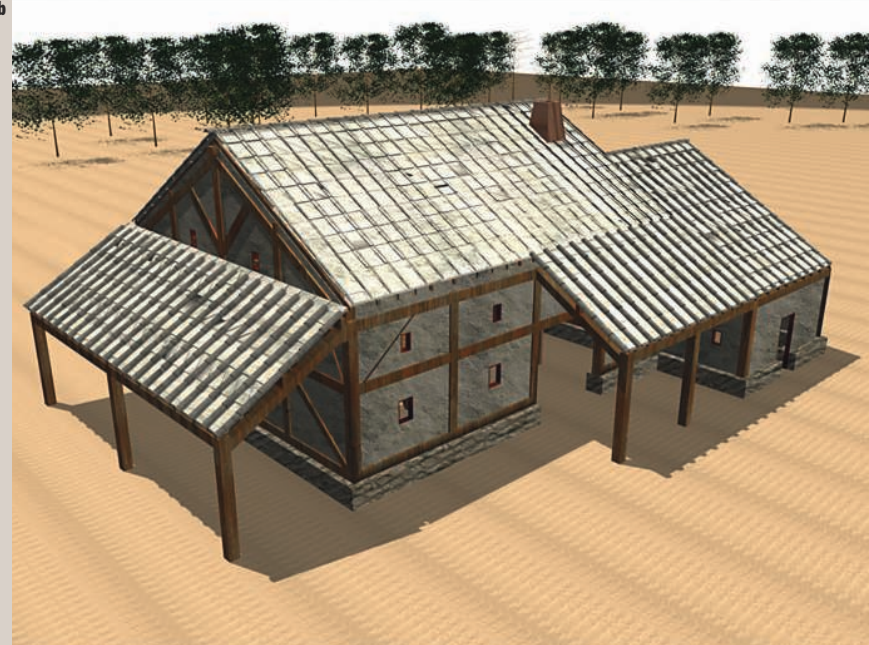




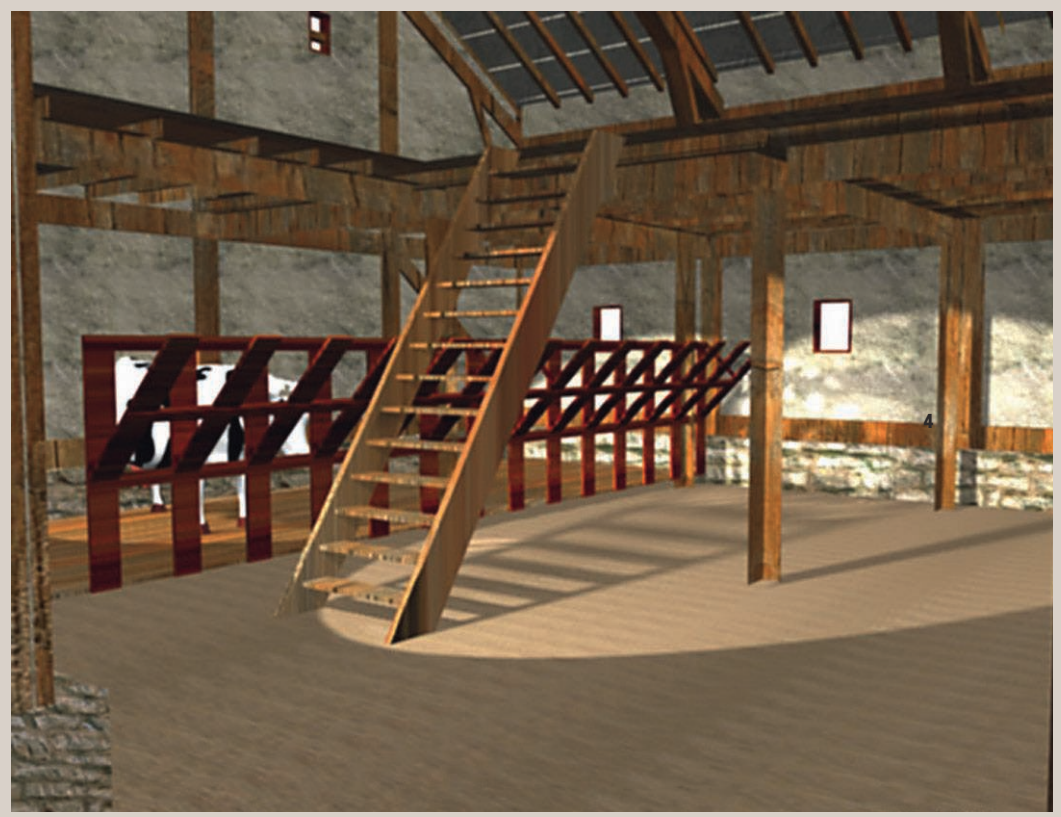

3.Proposition de restitution de l'étable.Cette image permet d'évoquer l'aspect probable de la partie réservée à la stabulation des bovidés. Séparée du reste de la grange par un râtelier, elle serait surmontée par un grenier en mezzanine destiné au stockage du fourrage.

a été dicté par le respect du module de base, en accord avec les proportions préconisées par les auteurs antiques. La restitution de la charpente de la grange repose sur l'implantation des poteaux axiaux et sur le module qui devait rythmer le pan de bois des façades. La découverte de tuiles dans les niveaux d'abandon a motivé le choix de la couverture. Enfin, la reconstitution des extensions périphériques reprend le système constitutif de la grange. Pour l'ensemble du bâtiment, l'implantation et la dimension des ouvertures relèvent à la fois des observations de fouille, de la logique climatique locale et des indications fournies par les traités d'agriculture antiques.

Cet exercice de restitution $3 \mathrm{D}$ a été présenté en septembre 2004 dans le cadre de la table ronde d'Insming (Moselle) organisée par l'ADRAL

(Association de recherche et de développement de l'archéologie en Lorraine) sur le thème «Restitution des bâtiments ruraux lorrains d'après les traces archéologiques : certitudes et incertitudes» (inédit). Certaines réactions de rejet ont permis d'appréhender combien il est important d'insister sur le fait que cette démarche n'est pas destinée à imposer une vision unique d'une réalité restituée. L'exercice soulève de nombreuses questions et oblige à proposer des réponses concrètes. Cependant, le résultat se doit d'être considéré comme la mise en image d'une hypothèse archéologique poussée à son terme, la création d'un support destiné à la réflexion et à l'ouverture des débats.

Cette première expérience positive s'est avérée riche en enseignements et a fait évoluer notre façon d'appréhender les fouilles suivantes. Ainsi, en 20082009, à l'occasion de la fouille des bains de la villa de Damblain (Vosges), la réflexion sur les restitutions des élévations a été ébauchée dès la phase de travail sur le terrain. Elle a permis d'attirer l'attention sur de nombreux indices, parfois ténus, et de porter les efforts de recherche sur certains matériaux parfois négligés, à l'exemple des enduits peints. Dès la phase de post-fouille, un travail commun de recherche mené avec les différents spécialistes a permis d'esquisser des propositions de restitution des élévations. Ce travail préparatoire a conduit naturellement à la mise en place d'un projet de restitution ${ }_{3} \mathrm{D}$ animée, actuellement réalisé en collaboration avec le SRA lorraine, l'Inrap et le CRAI (laboratoire de l'École nationale supérieure d'architecture de Nancy). Au terme de ce projet, l'outil de réflexion archéologique rejoindra la cause de la valorisation et de la communication auprès des spécialistes et du grand public.

La restitution $3 \mathrm{D}$ et l'étude architecturale des bâtiments à parois rejetées en région Centre aux $\mathrm{II}^{\mathrm{e}}-\mathrm{I}^{\mathrm{er}} \mathrm{S}$. avant notre ère

Gaëlle Robert, Inrap, UMR 7324 « Cités, Territoires, Environnement et Sociétés »

Nicolas Holzem, Inrap

La multiplication des fouilles en région Centre depuis une dizaine d'années sur la période du second âge du Fer a permis la découverte de très nombreux bâtiments datés des $\mathrm{II}^{\mathrm{e}} \mathrm{I}^{\mathrm{er}}$ siècles avant notre ère (Robert, 2009). Cette masse de données a conduit les archéologues spécialistes de la période à s'intéresser à l'architecture des bâtiments. La majorité de leurs plans, quadrangulaires avec quatre gros poteaux porteurs, semblent relativement homogènes. Ils présentent cependant des variations et un tableau typologique a pu être dressé à partir des découvertes du centre-ouest de la France pour cette période (Maguer, Robert, à paraître). Ainsi, les bâtiments d'habitation montrent le plus souvent un plan à quatre ou six poteaux de grandes dimensions, formant le module porteur de la construction. Des accès sont parfois marqués par une ou deux paires de poteaux moins profondément ancrés, disposés à l'extérieur du module porteur. Des traces de parois sont quelquefois observées, sous le forme de tranchées de fondation peu larges et peu profondes et/ou de trous de poteau. La surface réelle de ces bâtiments dépasse ainsi en général largement celle du module porteur, mais elle est souvent difficile à estimer.

Ce type de plan est désormais bien identifié dans la région. Toutefois, des recherches récemment publiées dans l'est de la France (Laurelut et al., 2005) ont permis d'observer une variation : le plan est toujours simple, carré ou rectangulaire à poteaux plantés, mais les supports du module porteur peuvent présenter une inclinaison légère, en moyenne de $10^{\circ}$ vers l'intérieur, comme sur le site de "Champ Chardon » ${ }^{\mathbf{1}}$ à Tours (Indre-et-Loire) [ill. 1]. Une dizaine de plans de ce type sont inventoriés dans le Centre, tous datés du $\mathrm{I}^{\mathrm{er}}$ siècle avant notre ère. Cette découverte fait désormais l'objet d'un intérêt particulier pour mieux comprendre les raisons de cette évolution du mode de construction.
1. Responsable scientifique : A. Couderc. 